\title{
Classification of Cancerous Skin using Artificial Neural Network Classifier
}

\author{
Mohammad Zakareya \\ Department of Information and \\ Communication Technology (ICT) \\ Mawlana Bhashani Science and \\ Technology University (MBSTU), \\ Santosh, Tnagail-1902, \\ Bangladesh
}

\author{
Mohammad Badrul Alam \\ Miah \\ Department of Information and \\ Communication Technology (ICT) \\ Mawlana Bhashani Science and \\ Technology University (MBSTU), \\ Santosh, Tnagail-1902, \\ Bangladesh
}

\author{
Md. Arafat Ullah \\ Department of Information and \\ Communication Technology (ICT) \\ Mawlana Bhashani Science and \\ Technology University (MBSTU), \\ Santosh, Tnagail-1902, \\ Bangladesh
}

\begin{abstract}
Cancer is one of the most hazardous diseases that cause death. However, if detected early this medical condition is not very prohibitive to defeat. The skin cancer is the anomalous growth of skin cells most often promotes on body apparent to the sunlight but can occur anywhere on the body. Skin cancer is the most common type of malignant tumor in both men and women. So, for the detection of cancer, image processing approaches play a paramount role. There are mainly four steps involved in the detection of skin cancer that are: Preprocessing, segmentation, feature extraction, and classification. The Neural network is used to classify images. It is an easy system rather than taking a biopsy from a doctor. The system consumes less time and gets the better result than the ordinary system.
\end{abstract}

\section{Keywords}

Skin Cancer, Feature extraction, and Neural Network

\section{INTRODUCTION}

Human Cancer is an intricate disease caused primarily by genetic inconstancy and accumulation of multiple molecular alternations [1][2]. During the clinical appearance, breast, skin, colon, a large percentage of prostate and ovarian cancer patients are hidden and metastatic colonies [3]. Among many types of cancer, Skin cancers are the most common form of cancers in human [4]. The human body has vast organ called skin. It separates the inward parts of the body and furthermore frames outer condition. The skin is a paramount organ that protects against body sensitivity, infection, pollution, odor, and it regulates body temperature. There are numerous side effects like skin drying, fatty acids, hair, and acne, which may cause or damage the skin surface. They might be caused by sensitivities, aggravations, hereditary issues and invulnerable framework issues. Every one of these side effects can bring about numerous diseases like skin break out, alopecia, dermatitis, and ringworms causing mutilation in look and feel of distinctive distressed. Diseases, for example, growth identified with Skin can be caused by numerous unmistakable reasons [5].

Unusual growth of skin cells due to skin cancer is often shown in the body's sunlight but can occur anywhere in the body. It promotes when an unrepaired DNA damage to the skin cells and mostly caused by ultraviolet radiation of the sun or tanning beds, trigger metamorphosis which leads to the skin cells multiply promptly and malignant tumors form. Some skin cancer can spread and cause confusion in the nearby tissue cells [6]. Sun is the most known cause of skin cancer. But it fully does not paraphrase that skin cancer usually develop on the skin exhibited to sunlight. Also, it can be exhibited to environmental threats, radiation analysis, and even legacy could play a role [7].

There are three types of skin cancer, they are- Basel cell cancer, squamous cell carcinoma, and malignant melanoma cancer. The first two does not grow quickly, but the third one growth quickly. Melanoma is much less common than skin cancer of basal cells and squamous cells, but it is much more dangerous than the other two types. However, it is much more hazardous if it is not found early. This is the reason for most of the deaths related to skin cancer $(75 \%)$ [8]. Bleeding, itching and a mass under the skin are other implications of cancerous change.

\section{RELATED WORK}

An automated system for detection and classification of one of the skin three types of skin cancerous proposed here: Melanoma, Basal cell carcinoma, Squamous cell carcinoma .There are a certain features of these types of skin cancers, which can be extracted using the proper feature extraction algorithm [9]. Various algorithms such as segmentation and characterization are used for the classification of chaotic skin jungle from a macroscopic image. A new system for characterizing digital images of skin lesions has been presented [10]. A scheme for automated detection of skin diseases by analyzing the texture recognition approaches based on gray level co-occurrence matrix (GLCM) is discussed here. The characteristic features of the test and the reference images and analyzed the skin diseases using texture analysis are extracted. Texture analysis is one of the fundamental aspects of human vision by which we differentiate between surfaces and objects [11].

Segmentation of the skin lesion of the surrounding skin in dermoscopic images using the segmentation algorithm of the neural network. Various segmentation approaches were used to the dermoscopic images to segment the skin lesions and calculated with 3 different metrics, such as sensitivity, accuracy and border error. Segmentation performance shows that Neural Network based lesion segmentation has high sensitivity, accuracy and less border error [12][13].

A study on past and present technology for skin cancer detection are given in detail with their relevant tools. Then it goes on discussing briefly about features, advantages or disadvantages of each of them, discussed the mathematics initial required to process the image of skin cancer lesion using the proposed scheme. [14]. A technique for the early 
detection of skin cancer problem is proposed. The diagnosis procedure for the treatment of malignant melanoma from other skin diseases, using digital image processing approaches and artificial neural networks. Dermoscopic images were composed and they are processed by numerous Image processing approaches. The cancerous region is separated from the healthy skin by the method of segmentation. [15]. The detection of melanoma based on region growing segmentation and the ABCD rule used for the detection of malignancy of brunette skin lesion is discussed. [16]. A method for detecting the border and identifying the occurrence and propagation of cancer by analyzing the variations of the RGB spectrum of lesion skin images using

\section{Image Acquisition}

The system has been collected total 3113 skin cancer images that are cancer and normal image of skin from The International Skin Imaging Collaboration and national cancer institute. The system used skin images that are .jpeg file format.

\subsection{Image Preprocessing}

The pre-processing is a modification of the image data that removes unwanted malformations or enhances some image features paramount for further processing. First, the image is resized to adjust images so that it supports feature classification with accuracy. After that, images are converted from RGB (Red, Green, and Blue) to the grey level where the features are based on texture properties.

\subsection{Image Preprocessing}

Image Segmentation in the computer vision system is the technique of partitioning a digital image into multiple segments. The intent of segmentation is to simplify or modify the description of an image into more meaningful and not difficult to analyze. Image segmentation is typically used to finds objects and boundaries (lines, curves, etc.) in images. More precisely, the image segmentation is a technique for designating a label of every pixel that shares the same pixellike features like an image. In the proposed system, segmentation processes consist of different steps shown below: novel Six Sigma threshold and region connectivity concepts is presented in this paper[17]. An automated method for melanoma diagnosis is applied on a set of dermoscopy images. Multilayer perceptron classifier to classify based on the extracted characteristics or to classify the gray-level cooccurrences matrix (GLCM) and the melanocytic navigator and the malignant melanoma [18].

\section{PROPOSED METHODOLOGY}

Fig 1. Shows the proposed methodology of the system

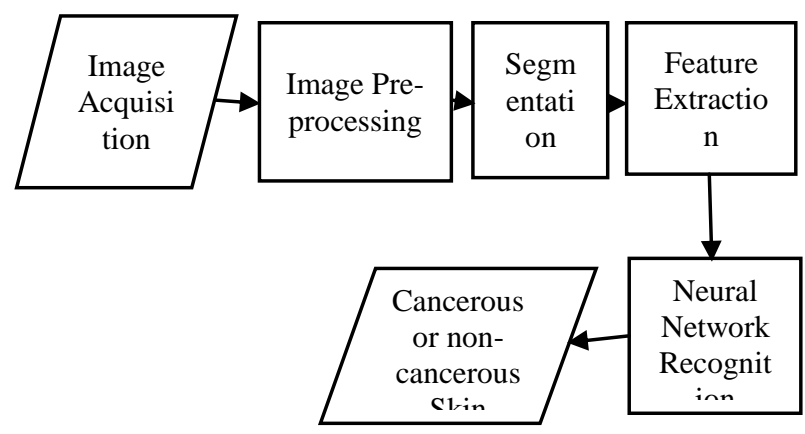

Fig 1: Block diagram of the proposed methodology

Figure 2 shows the Melanoma Skin Cancer

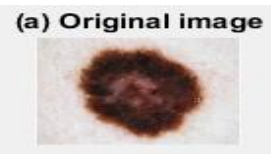

(c) Filtered Image

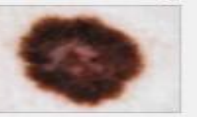

(e) Traced (b) Gray scale image

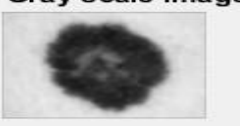

(d) Black and White

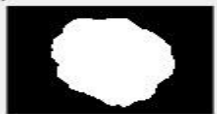

(f) Edges Only
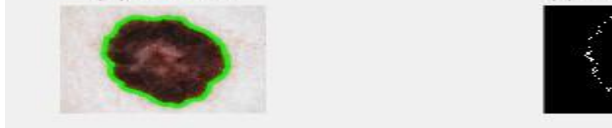

Fig 2: Melanoma Skin Cancer

Figure 3 shows the Basal Cell Carcinoma Skin Cancer (a) Original image

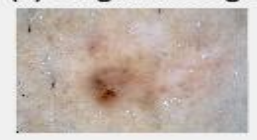

(c) Filtered Image

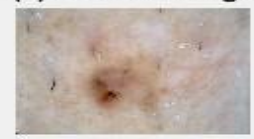

(e) Traced

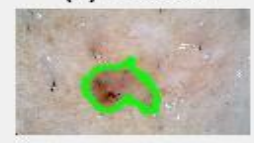

(b) Gray scale image

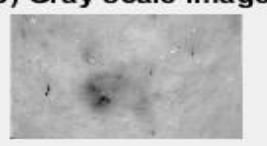

(d) Black and White

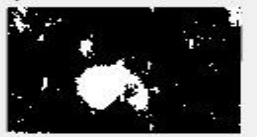

(f) Edges Only

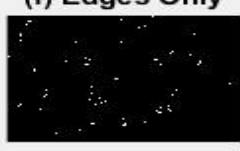

Fig 3: Basal Cell Carcinoma Skin Cancer 
Figure 4 shows the Squamous Cell Carcinoma Skin Cancer (a) Original image

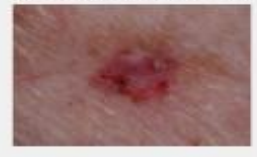

(c) Filtered Image

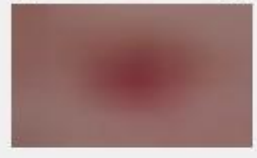

(e) Traced

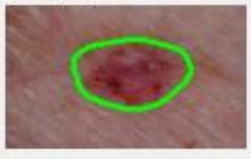

(b) Gray scale image

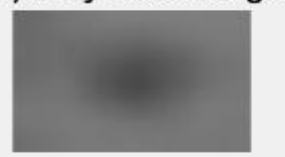

(d) Black and White

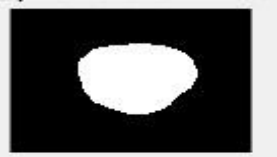

(f) Edges Only

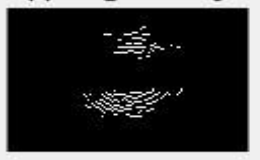

Fig 4: Squamous Cell Carcinoma Skin Cancer (a) Original image

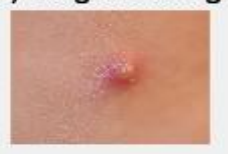

(c) Filtered Image

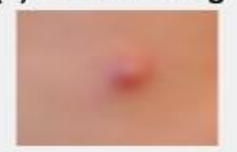

(e) Traced (b) Gray scale image

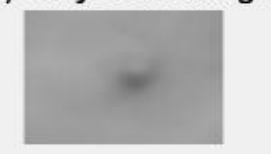

(d) Black and White

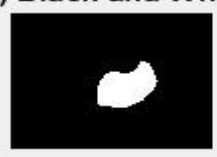

(f) Edges Only
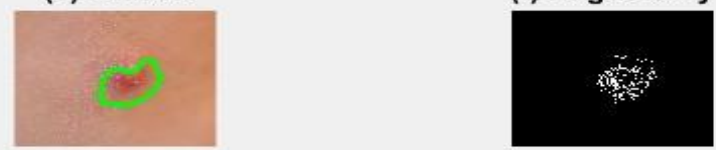

Fig 5: Normal Skin

\subsection{Feature Extraction}

Identifying the acknowledged qualities of texture in an image is the first step towards building mathematical models for texture. Because of the characteristic properties of an image intensity variation, the texture properties are usually visible for some internal physical characteristics.

Table 1. Shows the Formulas of texture properties

\begin{tabular}{|l|l|}
\hline Contrast & $\sum_{i, j}|i-j|^{2} p(i, j)$ \\
\hline Correlation & $\sum_{i, j} \frac{(i-? i)(j-? j) p(i, j)}{\sigma_{i}-\sigma_{j}}$ \\
\hline Homogeneity & $\sum_{i j} \frac{p(i, j)}{1+|i-j|}$ \\
\hline Energy & $\sum_{i, j} p(i, j)^{2}$ \\
\hline
\end{tabular}

Texture entails the spatial distribution of gray levels. Thus, two-dimensional histograms or co-occurrence matrices are acceptable texture analysis tools. Along with other statistical parameters such as entropy, centroid, solidity, mean, standard deviation, majorAxisLength, minorAxisLength, convexAera, eccentricity, orientation, filledArea, eulerNumber, equipDiameter, boundingBox, extent, perimeter, eucledian distance, city block distance, minkowski distance, hamming distance, momentum etc.

\subsection{Classification}

After the feature extraction process, these feature values are passed through the neural network to train up the system for classification purpose or detection purpose. The whole proposed training system for skin cancer detection consists of the following steps- Image Acquisition, Image Preprocessing, Segmentation, Feature Extraction, Neural Network Classification.

A neural network is employed for skin cancer detection. A multilayer feed forward neural network with directed learning method is more reliable and efficient for this purpose.

Figure 6 shows the Neural Network design of the proposed system:

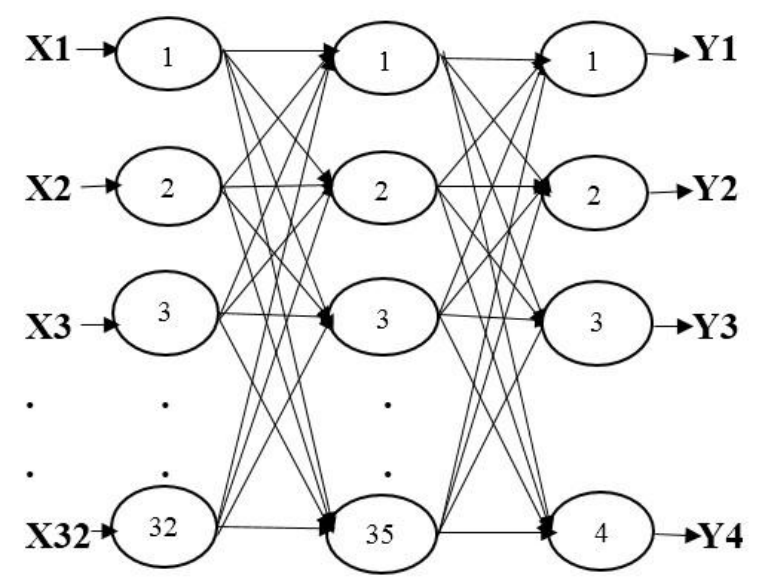

Fig 6: Neural Network Design of the system

Where $\mathrm{X} 1, \mathrm{X} 2, \mathrm{X} 3, \ldots \ldots ., \mathrm{X} 32$ input neurons, second part hidden layer, and final part Y1, Y2, Y3, Y4 are recognized output. Y1 means melanoma, Y2 means basal cell carcinoma, Y3 means squamous cell carcinoma skin cancer, and Y4 means normal skin.

Table 2: Shows the Initialize value of Neural Network parameters

\begin{tabular}{|c|c|}
\hline Parameter & Value \\
\hline Hidden Layer & 65 \\
\hline Output Layer & 4 \\
\hline Learning Rate & 0.0001 \\
\hline $\begin{array}{c}\text { Total number of } \\
\text { epochs }\end{array}$ & 1000 \\
\hline Performance Goal & 0.00000001 \\
\hline Momentum & 0.90 \\
\hline
\end{tabular}




\section{RESULT ANALYSIS AND DISCUSSION}

The MATLAB R2016a simulation tool is used for this algorithm. Most of all sampled images are collected from ISIC Archive and American Cancer Society. By using the sample image, the system extracts the value of images and detects and classifies the cancerous and non-cancerous skin.

Table 3: Shows the Test with cancerous skin images

\begin{tabular}{|l|l|l|l|l|}
\hline $\begin{array}{l}\text { Image } \\
\text { Description }\end{array}$ & $\begin{array}{l}\text { No. of } \\
\text { Image }\end{array}$ & $\begin{array}{l}\text { Successfully } \\
\text { Detection }\end{array}$ & $\begin{array}{l}\text { Accuracy } \\
(\%)\end{array}$ & $\begin{array}{l}\text { Error } \\
(\%)\end{array}$ \\
\hline $\begin{array}{l}\text { Skin Cancer- } \\
1\end{array}$ & 30 & 30 & 100 & 0 \\
\hline $\begin{array}{l}\text { Skin Cancer- } \\
2\end{array}$ & 32 & 31 & 96.87 & 3.13 \\
\hline $\begin{array}{l}\text { Skin Cancer- } \\
3\end{array}$ & 30 & 29 & 96.66 & 3.34 \\
\hline Total & 92 & 90 & 97.84 & 2.15 \\
\hline
\end{tabular}

Where Skin Cancer-1 means melanoma, Skin Cancer-2 means Basal Cell Carcinoma, Skin Cancer-3 means Squamous Cell Carcinoma Skin cancer.

Graph 1 shows the success rate for Cancerous skin images:

\section{Success Rate for Cancerous Skin Images}

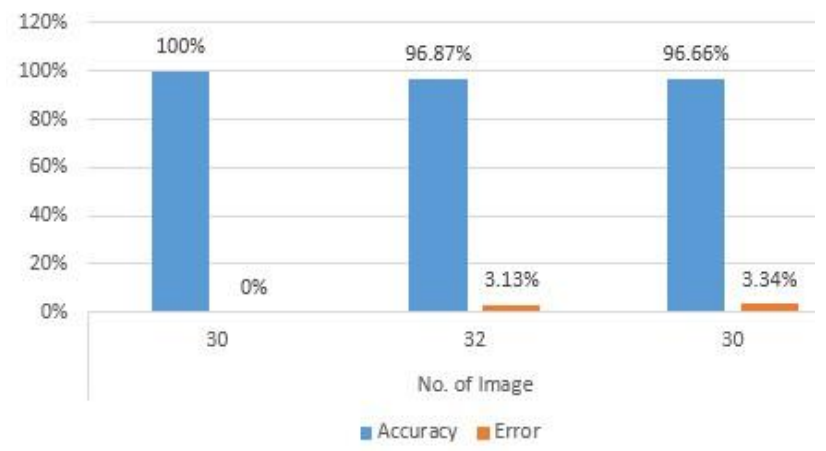

Graph 1: Image Number Vs Success Rate Cancerous Skin Images

Table 4: Shows the Test with Normal skin images

\begin{tabular}{|c|c|c|c|c|}
\hline $\begin{array}{c}\text { Image } \\
\text { Description }\end{array}$ & $\begin{array}{c}\text { No. of } \\
\text { Image }\end{array}$ & $\begin{array}{c}\text { Successfully } \\
\text { Detection }\end{array}$ & $\begin{array}{c}\text { Accuracy } \\
(\boldsymbol{\%})\end{array}$ & $\begin{array}{c}\text { Error } \\
(\boldsymbol{\%})\end{array}$ \\
\hline $\begin{array}{c}\text { Normal Skin } \\
\text { Image-1 }\end{array}$ & 10 & 10 & 100 & 0 \\
\hline $\begin{array}{c}\text { Normal Skin } \\
\text { Image-2 }\end{array}$ & 14 & 13 & 92.85 & 7.15 \\
\hline $\begin{array}{c}\text { Normal Skin } \\
\text { Image-3 }\end{array}$ & 5 & 5 & 100 & 0 \\
\hline $\begin{array}{c}\text { Normal Skin } \\
\text { Image-4 }\end{array}$ & 8 & 8 & 100 & 0 \\
\hline Total & 33 & 32 & 98.21 & 1.78 \\
\hline
\end{tabular}

Graph 2 shows the success rate for normal skin images:

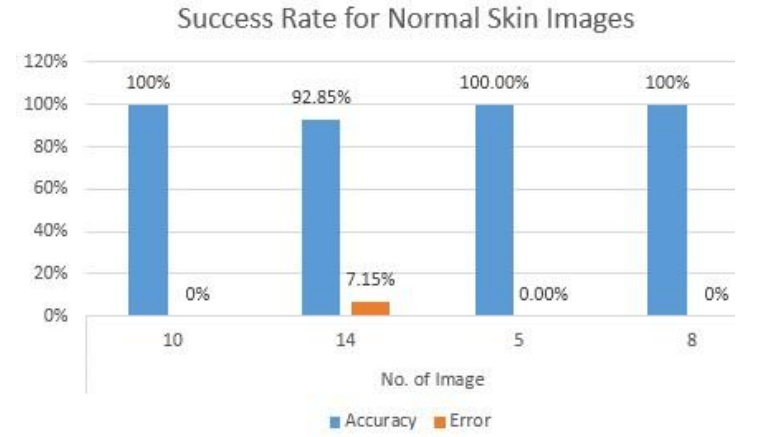

Graph 2: Image Number Vs Success Rate Normal Skin Images

\section{CONCLUSION}

Skin cancer is one kind of hazardous diseases, so it is necessary to detect early stages. In our proposed method chase approaches in which the first step is binary thresholding, and then feature extraction, and then these features are used to train up the neural network and test the neural network. The proposed system successfully detects and classifies the skin cancer from images. At the end of the system, you can say that the system achieves its expected expectations. The proposed system test 92 types of images and obtains the result where the overall success rate of the system is $97.84 \%$ which meet the expectation of the system. In future, this technique can be used in the detection of brain tumor, breast cancer, prostate cancer, lung cancer etc.

\section{ACKNOWLEDGMENTS}

We would like to express our deepest gratitude to Mohammad badrul Alam Miah, our thesis supervisor, for his guidance, enthusiastic encouragement and useful critiques of this thesis work. Besides my advisor, I would like to thank the rest my supervisory committee members, parents and friends.

\section{REFERENCES}

[1] Hanahan, D., \& Weinberg, R. A. (2011). Hallmarks of cancer: the next generation. cell, 144(5), 646-674.

[2] Bhuiyan, M. A. H., Ibrahim Azad, M., \& Uddin, K. (2013). Image processing for skin cancer features extraction. International Journal of Scientific \& Engineering Research, 4(2), 1-6.

[3] Menon, U., \& Jacobs, I. J. (2000). Recent developments in ovarian cancer screening. Current Opinion in Obstetrics and Gynecology, 12(1), 39-42.

[4] Kopf, A. W., Salopek, T. G., Slade, J., Marghoob, A. A., \& Bart, R. S. (1995). Approaches of cutaneous examination for the detection of skin cancer. Cancer, 75(S2), 684-690.

[5] Farooq, M. A., Azhar, M. A. M., \& Raza, R. H. (2016, October). Automatic Lesion Detection System (ALDS) for Skin Cancer Classification Using SVM and Neural Classifiers. In Bioinformatics and Bioengineering (BIBE), 2016 IEEE 16th International Conference on (pp. 301-308). IEEE.

[6] Saha, S., \& Gupta, R. (2013). An Automated Skin Lesion Diagnosis by using Image Processing Approaches. International Journal on Recent and Innovation Trends in Computing and Communication, 2(5), 1081-1085. 
[7] Zalaudek, I., Lallas, A., Moscarella, E., Longo, C., Soyer, H. P., \& Argenziano, G. (2013). The dermatologist's stethoscope - traditional and new applications of dermoscopy. Dermatology practical \& conceptual, 3(2), 67.

[8] Lu, C., Mahmood, M., Jha, N., \& Mandal, M. (2013) Automated segmentation of the melanocytes in skin histopathological images. IEEE journal of biomedical and health informatics, 17(2), 284-296.

[9] Maurya, R., Singh, S. K., Maurya, A. K., \& Kumar, A. (2014, March). GLCM and Multi Class Support vector machine based automated skin cancer classification. In Computing for Sustainable Global Development (INDIACom), 2014 International Conference on (pp. 444-447). IEEE.

[10] Messadi, M., Cherifi, H., \& Bessaid, A. (2014). Segmentation and $\mathrm{ABCD}$ rule extraction for skin tumors classification. Journal of Convergence Information Technology, 9(2), 21.

[11] Raja, C. V. J., \& Jeyaprakash, M. (2014). Skin Disease Diagnosis Using Texture Analysis. Sethu Institute of Technology and Chettinad College of Engg \& Tech, India, International Journal of Advanced Research in Computer Scienceand Software Engineering India, 4(1).

[12] Goel, R., \& Singh, S. (2015). Skin Cancer Detection using GLCM Matrix Analysis and Back Propagation Neural Network Classifier. International Journal of Computer Applications, 112(9).
[13] Al-Amin, M., Alam, M. B., \& Mia, M. R. (2015). Detection of Cancerous and Non-cancerous Skin by using GLCM Matrix and Neural Network Classifier. International Journal of Computer Applications, 132(8), 44.

[14] Ramteke, N. S., \& Jain, S. V. (2013). Analysis of Skin Cancer Using Fuzzy and Wavelet Technique-Review \& Proposed New Algorithm. International Journal of Engineering Trends and Technology (IJETT), 4(6), 2555-2566.

[15] Rani, N., Nalam, M., \& Mohan, A. (2014). Detection of Skin Cancer Using Artificial Neural Network. International Journal of Innovations \& Advancement in Computer Science IJIACS, 2(1).

[16] Smaoui, N., \& Bessassi, S. (2013). A developed system for melanoma diagnosis. International Journal of Computer Vision and Signal Processing, 3(1), 10-17.

[17] Sethumadhavan, G., \& Sankaran, S. (2009, June). Border detection and cancer propagation on spectral bands of malignant melanoma using six sigma threshold In Computer and Information Science, 2009. ICIS 2009. Eighth IEEE/ACIS International Conference on (pp. 586592). IEEE.

[18] Sheha, M. A., Mabrouk, M. S., \& Sharawy, A. (2012). Automatic detection of melanoma skin cancer using texture analysis. International Journal of Computer Applications, 42(20), 22-26. 